\title{
Antimicrobial Resistance Among Pathogens Causing Bloodstream Infections: A Multicenter Surveillance Report Over 20 Years (1998-2017)
}

\author{
Zhen Zhang', Ziyong Sun ${ }^{2}$, Lei Tian $^{2}$ \\ 'Department of Pharmacy, Tongji Hospital, Tongji Medical College, Huazhong University of Science and Technology, Wuhan, Hubei Province, People's \\ Republic of China; ${ }^{2}$ Department of Clinical Laboratory, Tongji Hospital, Tongji Medical College, Huazhong University of Science and Technology, \\ Wuhan, Hubei Province, People's Republic of China
}

Correspondence: Lei Tian, Department of Clinical Laboratory, Tongji Hospital, Tongji Medical College, Huazhong University of Science and Technology, Wuhan, Hubei Province, People's Republic of China, Email isol5189@I26.com

Purpose: Bloodstream infections (BSIs) are a common consequence of infectious diseases and cause high morbidity and mortality. Appropriate antibiotic use is critical for patients' treatment and prognosis. Long-term monitoring and analysis of antimicrobial resistance are important in guiding physicians to choose appropriate antibiotics and understand the changes in antimicrobial resistance and infection control. Here, we report a retrospective study on the trends of antimicrobial resistance in the common BSI-associated pathogens.

Methods: The identification of strains and antimicrobial susceptibility tests were performed in each anticipating hospital independently. Data from the Hubei Province Antimicrobial Resistance Surveillance System (HBARSS) from 1998 to 2017 were retrospectively analyzed using WHONET 5.6 software.

Results: Data from HBARSS (1998-2017) revealed that 40,518 Gram-positive bacteria and 26,568 Gram-negative bacteria caused BSIs, the most common of which were Staphylococcus aureus and Escherichia coli. Salmonella typhi was a predominant BSIassociated pathogen in 1998-2003. Antimicrobial susceptibility data showed that the resistance rates of E. coli and Klebsiella pneumoniae to cefotaxime were significantly higher than those to ceftazidime. The proportion of strains of special antimicrobial resistance phenotypes including difficult-to-treat resistance (DTR), carbapenem-resistant (CR), extended-spectrum cephalosporin resistant (ECR) and fluoroquinolone resistant (FQR) in E. coli was $0.18 \%, 0.26 \%, 13.95 \%, 22.78 \%$ while in K. pneumoniae was $11.95 \%, 14.00 \%, 31.91 \%$ and $11.40 \%$, respectively. In 2013-2017, K. pneumoniae showed resistance levels reaching $15.8 \%$ and $17.5 \%$ to imipenem and meropenem, respectively, and Acinetobacter baumannii showed high resistance rates ranging from 60 to $80 \%$ to common antibiotics. The detection rate of Salmonella typhi resistance to third-generation cephalosporins and fluoroquinolones was less than 5\%. Control of methicillin-resistant Staphylococcus aureus (MRSA) remains a major challenge, and in 2009-2017, the MRSA detection rate was $40-50 \%$.

Conclusion: Prevalence of CR K. pneumoniae has increased significantly in recent years. Resistance rates of A. baumannii to common antimicrobial agents have increased exponentially, reaching high levels. MRSA remains a challenge to control. For K. pneumoniae, DTR, CR, ECR and FQR were antimicrobial resistance phenotypes that could not be ignored while for E. coli DTR and CR were rare antimicrobial resistance phenotypes. CR K. pneumoniae, A. baumannii and MRSA present major challenges for controlling BSIs.

Keywords: Hubei Province Antimicrobial Resistance Surveillance System, HBARSS, antimicrobial resistance, bloodstream infections, methicillin-resistant Staphylococcus aureus, MRSA, carbapenem-resistant

\section{Introduction}

Antimicrobial resistance is a major health-related issue of global concern. Long-term monitoring of bacterial resistance is important for implementing effective control measures. ${ }^{1}$ Standardized and rational use of antibiotics can effectively reduce the occurrence of antimicrobial resistance. ${ }^{1}$ The report of antimicrobial resistance surveillance, including 
antimicrobial resistance genotypes and molecular epidemiological data, was very valuable for the global infection control strategy. $^{2}$ At present monitoring systems for antimicrobial resistance in China operate at the national, provincial and hospital levels. ${ }^{3}$

Hubei Province is located in central China and has 13 prefectural administrative regions under its jurisdiction. The Statistics Bureau of Hubei Province reports that in 1998, the permanent population of Hubei Province was 58.91 million, and by the end of 2018, this number was 59.17 million. Of this population, 35.68 million people lived in towns, and 23.49 million people lived in villages. ${ }^{4}$ The Hubei Province Antimicrobial Resistance Surveillance System (HBARSS) was founded in 1998 and initially consisted of 15 tertiary hospitals in different regions of Hubei Province. The number of network hospitals of HBARSS accounted for $13 \%$ of the general hospitals in Hubei province, while HBARSS covered $49.7 \%$ of the population in the whole province. Hospitals were added in 2003 and 2005, and 17 tertiary hospitals formed a monitoring network in Hubei Province. Since 2009, the monitoring network has been extended to secondary and tertiary hospitals across the entire province, and more than 50 hospitals have joined the monitoring network to date. The proportion of network hospitals from the registered hospitals for all of Hubei Province reached 14.45\% (50/346) in 2018.

Bloodstream infections (BSIs) are a major cause of morbidity and mortality in adults and children. ${ }^{5,6}$ Solid tumors, combined septic shock, indwelling catheters and continuous venovenous hemofiltration were independent risk factors affecting the prognosis of BSI patients. ${ }^{7}$ Appropriate use of antibiotics is critical for their treatment and prognosis. At present, China is one of the largest users of antibiotics worldwide, ${ }^{8}$ and antibiotic overuse remains a serious problem worldwide. ${ }^{9}$ Here, we report a 20-year analysis of HBARSS for 1998-2017. Our findings provide a reference for monitoring changes of antimicrobial resistance and management of antibiotics.

\section{Materials and Methods Study Design and Procedures}

To effectively analyze the accumulated susceptibility data and determine the trend in antimicrobial resistance for the major pathogens, only data from the initial 15 hospitals in 1998-2002, 16 hospitals in 2003-2004 and 17 hospitals in 2005-2017 were analyzed. Each network hospital independently cultured, identified and conducted antimicrobial susceptibility testing of the strains, and the data were submitted to HBARSS annually. Whonet 5.6 software was used to analyze the data of antimicrobial resistance tests.

Blood culturing was performed on patients who satisfied the clinical standards. ${ }^{10}$ Automated blood culture instruments, including the BD 9120, 9240 and FX 400 (BD Co., NJ, USA) or the 3D 120, 240 and 720 (Bio Mérieux, Lyon, France), were used in each hospital in the monitoring network. Strains were identified following each laboratory's protocol, which combined various automated instruments or an IVD-MALDI Biotyper (Bruker, Karlsruhe, Germany) with manual biochemical experiments. Either the disk-diffusion method or an automated instrument was used for the antimicrobial susceptibility tests. From 1998-2010, all hospitals used the disk-diffusion method for antimicrobial susceptibility testing. From 2011-2017, six hospitals used automated instruments, and 11 used the disk-diffusion method. Automated instruments for antimicrobial sensitivity testing included the Vitek-2 Compact system (Bio Mérieux, Lyon, France) and the domestic antimicrobial sensitivity testing system (Dier, Zhuhai, China). Antimicrobial susceptibility tests were performed strictly in accordance with Clinical Laboratory Standards Institute (CLSI) standards. Each hospital routinely carried out indoor quality control and participated in the External Quality Assessment of the Ministry of Health of China. Laboratory quality control experiments strictly followed the CLSI guidelines of the corresponding year, and standard strains were tested once weekly.

Because CoNS, Corynebacterium, Bacillus, Propionibacterium and other potential skin contaminants frequently contaminate blood cultures, whether these organisms were colonizing, pathogenic or contaminating bacteria was determined from the available clinical data. ${ }^{11}$

\section{Definition of Specific Antimicrobial Resistance Phenotypes}

In this study difficult-to-treat resistance (DTR) was defined as resistant in vitro to all ß-lactam categories, including carbapenems and fluoroquinolones. Carbapenem resistant (CR) was defined as resistant in vitro to imipenem and 
meropenem. Extended-spectrum cephalosporin resistant (ECR) was defined as resistant in vitro to ceftazidime, cefotaxime and cefepime. Fluoroquinolone resistant (FQR) was defined as resistant in vitro to ciprofloxacin and levofloxacin.

\section{Statistical Analysis}

Data were analyzed using WHONET 5.6 software. When multiple specimens were collected from the same patient simultaneously, only the first isolate of a given species from a patient was analyzed according to CLSI M-39. ${ }^{12}$ Interpretation criteria for the antimicrobial susceptibility results were based on CLSI 2020 Guidelines. ${ }^{13}$

\section{Ethical Statement}

The study protocol was approved by the Tongji Hospital ethics committee for research in health. The Tongji Hospital ethics committee also approved the waiver of informed consent to participate in this study due to its retrospective design. All patient data were anonymous prior to the analysis.

\section{Results}

\section{Distribution of Pathogenic Bacteria}

From 1998-2017, 40,518 Gram-positive bacterial strains and 26,568 Gram-negative bacterial strains were isolated from BSIs via HBARSS. The ratio of Gram-positive to Gram-negative bacteria was approximately 3:2 (Figure 1). The most common Gram-positive bacteria were coagulase-negative Staphylococcus (CoNS), Staphylococcus (S.) aureus, Streptococcus viride, Enterococcus faecalis and Enterococcus faecium $(\mathrm{N}>1000)$, while Escherichia (E.) coli, Klebsiella (K.) pneumoniae, Salmonella (S.) typhi, Pseudomonas (P.) aeruginosa, Stenotrophomonas (S.) maltophilia and Acinetobacter (A.) baumannii $(\mathrm{N}>1000)$ were the most common Gram-negative bacteria.

\section{Antimicrobial Susceptibility of Gram-Negative Bacteria}

Both $E$. coli and $K$. pneumoniae showed higher resistance to the third-generation cephalosporin, cefotaxime, than to ceftazidime. The resistance rates of $E$. coli to ceftazidime and cefotaxime were $10.5-30.1 \%$ and $31.75-67.3 \%$, respectively, whereas those of $K$. pneumoniae were $24-31.6 \%$ and $41.7-49.7 \%$, respectively. The resistance rate of $E$. coli to fluoroquinolones was significantly higher than that of $K$. pneumoniae. The resistance rates of $E$. coli to ciprofloxacin and levofloxacin were $47.3-55.6 \%$ and $45.2-52.8 \%$, respectively, and those of $K$. pneumoniae were $18.1-27.7 \%$ and $11.9-$ $25.5 \%$, respectively. The resistance rate of $K$. pneumoniae to carbapenems was significantly higher than that of E. coli. The resistance rates of $K$. pneumoniae to imipenem and meropenem were $2.4-15.8 \%$ and $1.8-17.5 \%$, respectively, whereas those of E. coli were $0.8-2.3 \%$ and $0.8-1.3 \%$, respectively (Table 1). S. typhi showed resistance to thirdgeneration cephalosporins and fluoroquinolones, but the resistance rate was less than $6 \%$. The resistance rate of $S$. typhi to ampicillin increased significantly from $6.9 \%$ in $1998-2002$ to $38.5 \%$ in $2013-2017$ (Table 2).

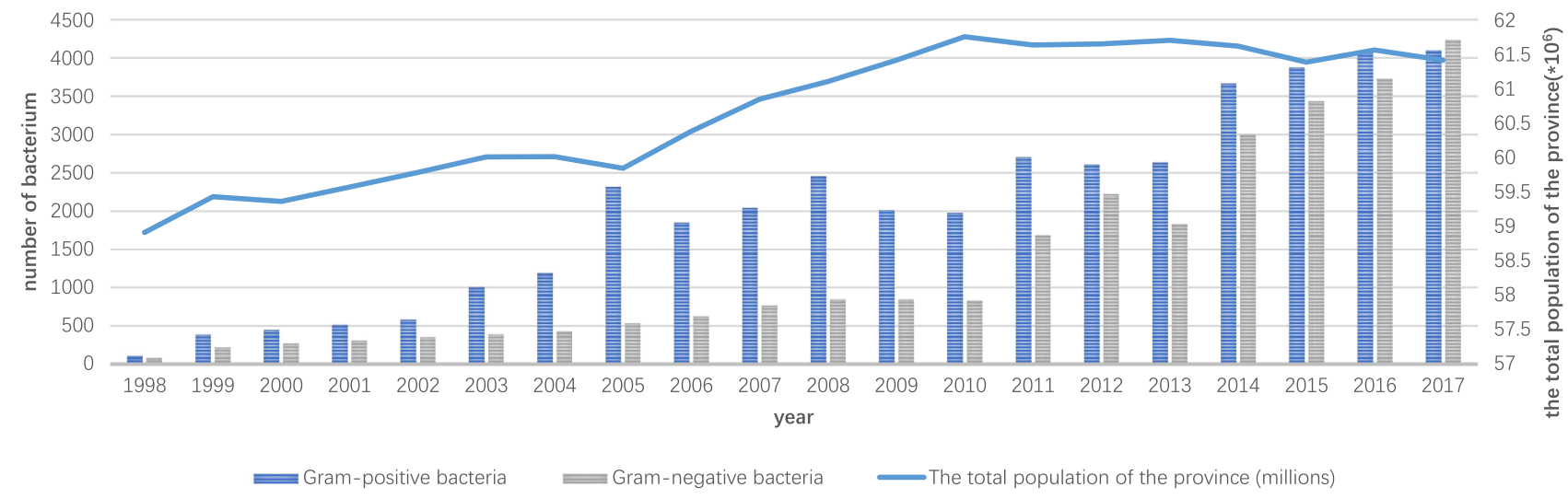

Figure I Demographic data and pathogens of BSI in Hubei Province. 
Table I Resistance Rates of Escherichia coli and Klebsiella pneumoniae to the Common Antibiotics

\begin{tabular}{|c|c|c|c|c|c|c|c|c|c|c|c|c|c|c|c|c|}
\hline & \multicolumn{8}{|c|}{ Escherichia coli } & \multicolumn{8}{|c|}{ Klebsiella pneumoniae } \\
\hline & \multicolumn{2}{|c|}{ 1998-2002 } & \multicolumn{2}{|c|}{ 2003-2007 } & \multicolumn{2}{|c|}{$2008-2012$} & \multicolumn{2}{|c|}{$2013-2017$} & \multicolumn{2}{|c|}{ 1998-2002 } & \multicolumn{2}{|c|}{ 2003-2007 } & \multicolumn{2}{|c|}{ 2008-20I2 } & \multicolumn{2}{|c|}{$2013-2017$} \\
\hline & $\mathbf{n}$ & $\mathbf{R}(\%)$ & $\mathbf{n}$ & $\mathbf{R}(\%)$ & $\mathbf{n}$ & $\mathbf{R}(\%)$ & $\mathbf{n}$ & $\mathbf{R}(\%)$ & $\mathbf{n}$ & $\mathbf{R}(\%)$ & $\mathbf{n}$ & $\mathbf{R}(\%)$ & $\mathbf{n}$ & $\mathbf{R}(\%)$ & $\mathbf{n}$ & $\mathbf{R}(\%)$ \\
\hline Penicillins & & & & & & & & & & & & & & & & \\
\hline $\begin{array}{l}\text { Piperacillin (100ug) } \\
\beta \text {-Lactam combination agents }\end{array}$ & 343 & 71.1 & 948 & 79.4 & 2652 & 81.1 & 4883 & 79 & 90 & 56.7 & 278 & 48.9 & 738 & 49.7 & 1960 & 52 \\
\hline Ampicillin clavulanic acid (20/lOug) & 328 & 22 & 859 & 17.2 & 2468 & 17.7 & 4968 & 14.1 & 95 & 28.4 & 262 & 17.6 & 701 & 19.5 & 2005 & 29.6 \\
\hline Cefoperazone sulbactam (75/75ug) & l & l & 930 & 9.2 & 2479 & 5.5 & 2962 & 7.6 & 48 & 18.8 & 259 & 12 & 688 & 9.3 & 1260 & 30.8 \\
\hline $\begin{array}{l}\text { Piperacillin Tazobactam (100/IOug) } \\
\text { Cephems (including cephalosporins I, II, III, } \\
\text { and IV) }\end{array}$ & l & 1 & 1 & l & 2062 & 4.7 & 6619 & 3.7 & 1 & 1 & 120 & 20.8 & 587 & 9.9 & 2560 & 20.4 \\
\hline Cefuroxime (30ug) & 304 & 31.9 & 893 & 57.7 & 2520 & 68.4 & 5228 & 61.5 & 82 & 42.7 & 270 & 42.2 & 715 & 44.1 & 1889 & 45.8 \\
\hline Ceftazidime (30ug) & 352 & 10.5 & 1015 & 17.7 & 2904 & 30.1 & 7137 & 26.6 & 96 & 24 & 290 & 25.9 & 810 & 27.7 & 2680 & 31.6 \\
\hline Cefotaxime (30ug) & 356 & 31.7 & 989 & 56.6 & 2611 & 67.3 & 4467 & 62.5 & 97 & 42.3 & 288 & 41.7 & 695 & 44.3 & 1814 & 49.7 \\
\hline Cefepime (30ug) & I & I & 973 & 33.4 & 2812 & 48.8 & 7122 & 41.3 & 38 & 39.5 & 279 & 29 & 780 & 30.1 & 2688 & 33.6 \\
\hline $\begin{array}{l}\text { Cefoxitin (30ug) } \\
\text { Monobactams }\end{array}$ & l & 1 & 824 & 12.7 & 2393 & 11.5 & 5136 & 7.5 & I & 1 & 239 & 18 & 674 & 13.9 & 1866 & 25.2 \\
\hline $\begin{array}{l}\text { Aztreonam (30ug) } \\
\text { Carbapenems }\end{array}$ & 329 & 14.6 & 969 & 25.6 & 2684 & 40.9 & 5704 & 37 & 90 & 34.4 & 278 & 25.9 & 745 & 30.2 & 2283 & 35.2 \\
\hline Imipenem (IOug) & 341 & 2.3 & 1011 & 1.8 & 2850 & $\mathrm{I}$ & 7173 & 0.8 & 88 & 9.1 & 289 & 2.4 & 802 & 3.7 & 2685 & 15.8 \\
\hline Meropenem (IOug) & l & 1 & 366 & 0.8 & 1988 & 1.2 & 6331 & 1.3 & 1 & 1 & 113 & 1.8 & 567 & 4.4 & 2372 & 17.5 \\
\hline Aminoglycosides & & & & & & & & & & & & & & & & \\
\hline Amikacin (30ug) & 352 & 9.1 & 964 & 6 & 2870 & 6.2 & 6900 & 2.4 & 95 & 23.2 & 280 & 6.8 & 806 & 5.7 & 2620 & 12.7 \\
\hline Gentamicin (IOug) & 352 & 47.4 & 992 & 55.1 & 2775 & 52.5 & 7152 & 39.5 & 96 & 35.4 & 285 & 23.2 & 793 & 25.7 & 2694 & 28.8 \\
\hline Fluoroquinolones & & & & & & & & & & & & & & & & \\
\hline Ciprofloxacin (5ug) & 360 & 52.8 & 973 & 55.6 & 2448 & 54.3 & 7091 & 47.3 & 97 & 18.6 & 285 & 19.3 & 722 & 18.1 & 2668 & 27.7 \\
\hline Levofloxacin (5ug) & l & 1 & 702 & 52.8 & 2190 & 54.6 & 6448 & 45.2 & l & l & 193 & 11.9 & 601 & 13.5 & 2511 & 25.5 \\
\hline Folate pathway antagonists & & & & & & & & & & & & & & & & \\
\hline Trimethoprim/sulfamethoxazole (1.25/23.75ug) & 351 & 72.1 & 980 & 72.2 & 2636 & 62.8 & 7101 & 56.6 & 95 & 44.2 & 288 & 46.9 & 723 & 38.7 & 2673 & 31.6 \\
\hline
\end{tabular}

Note: "l" indicated that the antibiotics had not been tested. 
Table 2 Resistance Rates of Salmonella typhi to the Common Antibiotics

\begin{tabular}{|c|c|c|c|c|c|c|c|c|}
\hline & \multicolumn{2}{|c|}{ 1998-2002 } & \multicolumn{2}{|c|}{ 2003-2007 } & \multicolumn{2}{|c|}{$2008-2012$} & \multicolumn{2}{|c|}{$2013-2017$} \\
\hline & $\mathbf{n}$ & $\mathbf{R}(\%)$ & $\mathbf{n}$ & $\mathbf{R}(\%)$ & $\mathbf{n}$ & $\mathbf{R}(\%)$ & $\mathbf{n}$ & $\mathbf{R}(\%)$ \\
\hline \multicolumn{9}{|l|}{ Penicillins } \\
\hline Ampicillin (IOug) & 202 & 6.9 & 116 & 15.5 & 69 & 26.1 & 52 & 38.5 \\
\hline \multicolumn{9}{|l|}{ Fluoroquinolones } \\
\hline Ciprofloxacin (5ug) & 194 & 4.1 & 119 & 4.2 & 66 & 3 & 54 & 3.7 \\
\hline Levofloxacin (5ug) & 34 & 0 & 65 & 1.5 & 59 & 0 & 37 & 2.7 \\
\hline \multicolumn{9}{|l|}{ Folate pathway antagonists } \\
\hline $\begin{array}{l}\text { Trimethoprim/sulfamethoxazole (I.25/ } \\
\text { 23.75ug) }\end{array}$ & 182 & 22 & 121 & 25.6 & 66 & 10.6 & 57 & 8.8 \\
\hline \multicolumn{9}{|l|}{ Cephems } \\
\hline Ceftazidime (30ug) & 192 & 3.6 & 109 & 1.8 & 62 & 3.2 & 42 & 0 \\
\hline Cefatriaxone (30ug) & 41 & 2.4 & 62 & 1.6 & 38 & 5.3 & 23 & 0 \\
\hline Cefotaxime (30ug) & 196 & 6.1 & 104 & 1.9 & 58 & 8.6 & 32 & 3.1 \\
\hline \multicolumn{9}{|l|}{ Phenicols } \\
\hline Chloramphenicol (30ug) & 83 & 6 & 68 & 7.4 & 33 & 9.1 & 41 & 7.3 \\
\hline
\end{tabular}

Most resistance rates of $P$. aeruginosa to common antibiotics were less than $30 \%$. The resistance rates of A. baumannii to common antimicrobial agents increased significantly from less than $50 \%$ in $2003-2007$ to $55-70 \%$ in 2008-2012 (except to cefoperazone sulbactam) and to 60-80\% in 2013-2017. From 1998 to 2017, the detection rates of extensively drug-resistant $A$. baumannii and $P$. aeruginosa were $34.38 \%$ (493/1434) and 7.45\% (140/1879), respectively (Table 3). S. maltophilia was not resistant to ceftazidime in 1998-2012 but then showed a resistance rate of $58.1 \%$ in 2013-2017 (Table 4).

\section{Epidemiology of Methicillin-Resistant Staphylococcus aureus (MRSA)}

The MRSA detection rate was $10-30 \%$ in $1998-2003$, which increased to $20-70 \%$ in $2004-2007$ and $40-50 \%$ in 2009 2017 (Figure 2). The resistance rate of MRSA to common antibiotics was significantly higher than that of MSSA. The resistance rate of MRSA to trimethoprim/sulfamethoxazole decreased significantly from $69.9 \%$ in $1998-2002$ to $3.8 \%$ in 2012-2017, and that of MSSA decreased significantly from 29.2\% in 2003-2007 to 3.3\% in 2013-2017 (Table 5).

\section{Distribution of Specific Antimicrobial Resistance Phenotypes}

For E. coli, DTR and CR were rare drug-resistant phenotypes, accounting for $0.18 \%(21 / 11597)$ and $0.26 \%(30 / 11597)$ respectively. While ECR and FQR were the main drug-resistant phenotypes, accounting for $13.95 \%(1618 / 11597)$ and $22.78 \%(2642 / 11597)$ respectively. But for $K$. pneumoniae, ECR were the most common drug-resistant phenotype, accounting for $31.91 \%(622 / 3949)$. The distribution proportion of DTR, CR and FQR was almost equal, accounting for $11.95 \%$ (233/3949), 14.00\% (273/3949) and 11.40\% (450/3949) respectively (Figure 3).

\section{Discussion}

Surveillance data during 1998-2017 in Hubei Province showed that the most common BSI-associated Gram-negative and Gram-positive bacteria were E. coli and $S$. aureus, respectively. This finding was consistent with that of the European Antimicrobial Resistance Surveillance Network (EARS-Net, formerly EARSS) report for 2002-2009 ${ }^{14}$ but differed from reports from Malawi, Africa, which showed that non-typhoid Salmonella, S. typhi and Streptococcus pneumoniae were the main BSI-associated pathogens. ${ }^{6}$ Our findings were consistent with the previous reports in China. A study on trends in antimicrobial resistance in BSI at the first affiliated hospital of Zhengzhou University in China indicated E. coli, K. pneumoniae, S. aureus, A. baumannii and P. aeruginosa were the most common pathogen. ${ }^{15}$

Our study showed that $S$. typhi was also a main BSI-associated pathogen in Hubei Province during 1998-2003. Typhoid fever is a poverty-related disease, mainly occurring in Africa and Asia, with a low incidence in economically 
Table 3 Resistance Rates of Acinetobacter baumannii and Pseudomonas aeruginosa to the Common Antibiotics

\begin{tabular}{|c|c|c|c|c|c|c|c|c|c|c|c|c|c|c|c|c|}
\hline & \multicolumn{8}{|c|}{ Acinetobacter baumannii } & \multicolumn{8}{|c|}{ Pseudomonas aeruginosa } \\
\hline & \multicolumn{2}{|c|}{$1998-2002$} & \multicolumn{2}{|c|}{ 2003-2007 } & \multicolumn{2}{|c|}{$2008-2012$} & \multicolumn{2}{|c|}{$2013-2017$} & \multicolumn{2}{|c|}{ 1998-2002 } & \multicolumn{2}{|c|}{ 2003-2007 } & \multicolumn{2}{|c|}{$2008-2012$} & \multicolumn{2}{|c|}{$2013-2017$} \\
\hline & $\mathbf{n}$ & R (\%) & $\mathbf{n}$ & $\mathbf{R}(\%)$ & $\mathbf{n}$ & $\mathbf{R}(\%)$ & $\mathbf{n}$ & R (\%) & $\mathbf{n}$ & $\mathbf{R}(\%)$ & $\mathbf{n}$ & $\mathbf{R}(\%)$ & $\mathbf{n}$ & R (\%) & $\mathbf{n}$ & $\mathbf{R}(\%)$ \\
\hline Penicillins & & & & & & & & & & & & & & & & \\
\hline $\begin{array}{l}\text { Piperacillin (100ug) } \\
\beta \text {-Lactam combinationagents }\end{array}$ & 9 & 22.2 & 64 & 50 & 341 & 69.5 & 760 & 78.8 & 99 & 24.2 & 189 & 29.1 & 473 & 23.7 & 1065 & 21.4 \\
\hline Cefoperazone sulbactam (75/75ug) & I & l & 62 & 12.9 & 299 & 24.4 & 643 & 63.1 & l & l & 1 & 1 & 1 & l & I & l \\
\hline $\begin{array}{l}\text { Piperacillin Tazobactam (100//0ug) } \\
\text { Cephems }\end{array}$ & l & I & 36 & 41.7 & 245 & 62 & 876 & 74.9 & I & I & 89 & 19.1 & 334 & 13.2 & 1046 & 15.6 \\
\hline Ceftazidime (30ug) & 10 & 30 & 68 & 42.6 & 351 & 63 & 879 & 76.5 & 100 & 26 & 191 & 18.8 & 471 & 21 & 1079 & 19 \\
\hline $\begin{array}{l}\text { Cefepime (30ug) } \\
\text { Carbapenems }\end{array}$ & 1 & 1 & 65 & 38.5 & 310 & 62.9 & 986 & 75.4 & 1 & 1 & 175 & 17.7 & 465 & 21.3 & 1081 & 19.9 \\
\hline Imipenem (10ug) & I & I & 68 & 23.5 & 334 & 61.1 & 989 & 74.4 & 95 & 16.8 & 189 & 21.2 & 473 & 23.7 & 1079 & 30.6 \\
\hline $\begin{array}{l}\text { Meropenem (IOug) } \\
\text { Aminoglycosides }\end{array}$ & I & I & 30 & 20 & 272 & 58.1 & 829 & 76.1 & 1 & 1 & 87 & 14.9 & 381 & 21.8 & 935 & 25.6 \\
\hline Amikacin (30ug) & 10 & 20 & 64 & 39.1 & 351 & 59.8 & 916 & 67.7 & 101 & 16.8 & 186 & 17.7 & 480 & 20.2 & 1063 & 14.3 \\
\hline Gentamicin (IOug) & 10 & 40 & 65 & 49.2 & 344 & 67.4 & 986 & 75.5 & 103 & 23.3 & 186 & 29.6 & 468 & 26.3 & 1075 & 21.2 \\
\hline Fluoroquinolones & & & & & & & & & & & & & & & & \\
\hline Ciprofloxacin (5ug) & 10 & 40 & 63 & 31.7 & 317 & 57.7 & 969 & 74.5 & 98 & 17.3 & 187 & 17.1 & 423 & 18.4 & 1069 & 17.5 \\
\hline Levofloxacin (5ug) & I & I & 46 & 28.3 & 273 & 57.9 & 928 & 69.6 & 1 & 1 & 107 & 21.5 & 326 & 13.8 & 939 & 17.3 \\
\hline Folate pathway antagonists & & & & & & & & & & & & & & & & \\
\hline Trimethoprim/sulfamethoxazole (1.25/23.75ug) & 8 & 25 & 68 & 50 & 268 & 63.4 & 962 & 74.3 & I & l & l & I & I & I & I & I \\
\hline
\end{tabular}

Note: "l" indicated that the antibiotics had not been tested. 
Table 4 Resistance Rates of Stenotrophomonas maltophilia to the Common Antibiotics

\begin{tabular}{|c|c|c|c|c|c|c|c|c|}
\hline & \multicolumn{2}{|c|}{ 1998-2002 } & \multicolumn{2}{|c|}{ 2003-2007 } & \multicolumn{2}{|c|}{ 2008-20 I } & \multicolumn{2}{|c|}{$2013-2017$} \\
\hline & $\mathbf{n}$ & $\mathbf{R}(\%)$ & $\mathbf{n}$ & $\mathbf{R}(\%)$ & $\mathbf{n}$ & $\mathbf{R}(\%)$ & $\mathbf{n}$ & $\mathbf{R}(\%)$ \\
\hline Fluoroquinolones & & & & & & & & \\
\hline Levofloxacin (5ug) & 16 & 6.2 & 191 & 1 & 266 & 1.9 & 520 & 4.2 \\
\hline Folate pathway antagonists & & & & & & & & \\
\hline $\begin{array}{l}\text { Trimethoprim/sulfamethoxazole ( } 1.25 / \\
\text { 23.75ug) }\end{array}$ & 38 & 10.5 & 243 & 9.5 & 268 & 22.4 & 531 & II.I \\
\hline Cephems & & & & & & & & \\
\hline Ceftazidime (30ug) & 41 & 0 & 171 & 0 & 102 & 0 & 365 & 58.1 \\
\hline Phenicols & & & & & & & & \\
\hline Chloramphenicol (30ug) & 24 & 0 & 90 & 0 & 19 & 0 & 259 & 5 \\
\hline
\end{tabular}

developed regions such as Europe and the United States. ${ }^{16-20}$ Typhoid fever is transmitted mainly through contaminated food and drinking water. ${ }^{21}$ The incidence of $S$. typhi-related BSIs in rural children was reported to be 2-3 times higher than that in urban children. ${ }^{22}$ The different incidences in different areas may be related to local medical and health conditions and vaccination rates. These factors may also have contributed to the high incidence in Hubei Province during 1998-2003. Reports from Africa suggested that S. typhi and non-S. typhi were consistently the most common pathogens of BSIs. ${ }^{6}$ Salmonella infections are frequently associated with human immunodeficiency virus infections, very young or elderly patients, clinical malaria and malnutrition, and can be fatal in up to $20-25 \%$ of patients. ${ }^{23,24}$ Reports from Africa showed that Salmonella was often resistant to first-line antibiotics such as chloramphenicol, sulfonamide and ampicillin. ${ }^{25,26}$ In our study, the resistance rate of $S$. typhi to ampicillin increased from $6.9 \%$ in $1998-2002$ to $38.5 \%$ in 2013-2017, and resistance rates to other antibiotics were lower than 10\% in 2013-2017. The significant increase of antimicrobial resistance to ampicillin might be related to the extensive use of ampicillin. Given the fact that the target of ampicillin is located in the cell wall, and human just lack the cell wall, the use of ampicillin for treatment is relatively safe, which leads to the extensive use of ampicillin in clinical treatment. Resistance to fluoroquinolones and thirdgeneration cephalosporins has also been reported in several African countries. ${ }^{27,28}$ Our data showed that $S$. typhi resistance to third-generation cephalosporins and fluoroquinolones has emerged, but in 1998-2017, the detection rate was less than $5 \%$. The disadvantages of this study was that we did not detect the antimicrobial resistance gene of

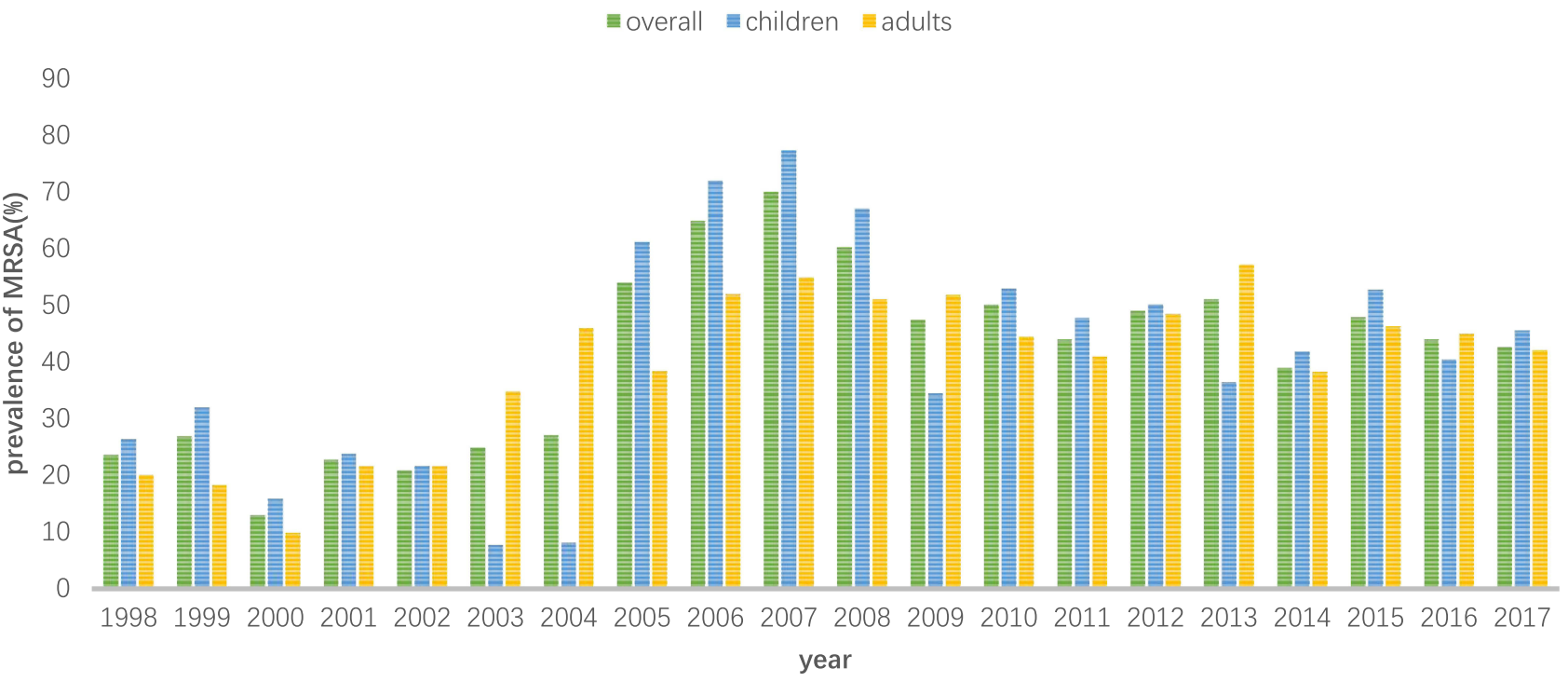

Figure 2 Prevalence of MRSA (\%) in adults ( $\geq 18$ years) and children ( $<18$ years). 
Table 5 Resistance Rates of MRSA and MSSA to the Common Antibiotics

\begin{tabular}{|c|c|c|c|c|c|c|c|c|c|c|c|c|c|c|c|c|}
\hline & \multicolumn{8}{|c|}{ MRSA } & \multicolumn{8}{|c|}{ MSSA } \\
\hline & \multicolumn{2}{|c|}{$1998-2002$} & \multicolumn{2}{|c|}{ 2003-2007 } & \multicolumn{2}{|c|}{$2008-2012$} & \multicolumn{2}{|c|}{$2013-2017$} & \multicolumn{2}{|c|}{$1998-2002$} & \multicolumn{2}{|c|}{ 2003-2007 } & \multicolumn{2}{|c|}{ 2008-20I 2} & \multicolumn{2}{|c|}{$2013-2017$} \\
\hline & $\mathbf{n}$ & R (\%) & $\mathbf{n}$ & R (\%) & $\mathbf{n}$ & R (\%) & $\mathbf{n}$ & R (\%) & $\mathbf{n}$ & R (\%) & $\mathbf{n}$ & R (\%) & $\mathbf{n}$ & $\mathbf{R}(\%)$ & $\mathbf{n}$ & R (\%) \\
\hline Aminoglycosides & & & & & & & & & & & & & & & & \\
\hline $\begin{array}{l}\text { Gentamicin (IOug) } \\
\text { Fluoroquinolones }\end{array}$ & 89 & 38.2 & 345 & 55.4 & 442 & 56.6 & 520 & 77.9 & 305 & 5.6 & 431 & 9.7 & 524 & 10.5 & 421 & 10.7 \\
\hline $\begin{array}{l}\text { Levofloxacin (5ug) } \\
\text { Folate pathway antagonists }\end{array}$ & I & I & l & 1 & 380 & 53.9 & 455 & 82.6 & 1 & 1 & 1 & 1 & 446 & 7.8 & 305 & 7.5 \\
\hline $\begin{array}{l}\text { Trimethoprim/sulfamethoxazole ( } 1.25 / 23.75 \text { ug) } \\
\text { Lincosamides }\end{array}$ & 83 & 69.9 & 379 & 64.9 & 435 & 41.1 & 504 & 3.8 & 300 & 26.7 & 449 & 29.2 & 501 & 22.2 & 397 & 3.3 \\
\hline $\begin{array}{l}\text { Clindamycin (2ug) } \\
\text { Macrolides }\end{array}$ & 72 & 61.1 & 388 & 67.5 & 462 & 63.6 & 514 & 55.3 & 265 & 30.6 & 446 & 28.7 & $54 I$ & 21.4 & 401 & 19.5 \\
\hline $\begin{array}{l}\text { Erythromycin (15ug) } \\
\text { Oxazolidinones }\end{array}$ & 90 & 85.6 & 393 & 89.1 & 461 & 85.7 & 526 & 72.4 & 313 & 62.3 & 451 & 54.8 & 540 & 54.1 & 422 & 46.2 \\
\hline $\begin{array}{l}\text { Linezolid (30ug) } \\
\text { Glycopeptides }\end{array}$ & I & I & 1 & 1 & I & 1 & 487 & 0 & 1 & 1 & 1 & 1 & I & I & 376 & 0 \\
\hline Vancomycin (30ug) & 90 & 0 & 393 & 0 & 370 & 0 & 475 & 0 & 319 & 0 & 454 & 0 & 444 & 0 & 326 & 0 \\
\hline Teicoplanin (30ug) & I & I & 360 & 1.1 & 434 & 1.2 & 491 & 0.2 & 1 & I & 424 & 0 & 476 & 0.2 & 329 & 0 \\
\hline
\end{tabular}

Note: "N" indicated that the antibiotics had not been tested. 


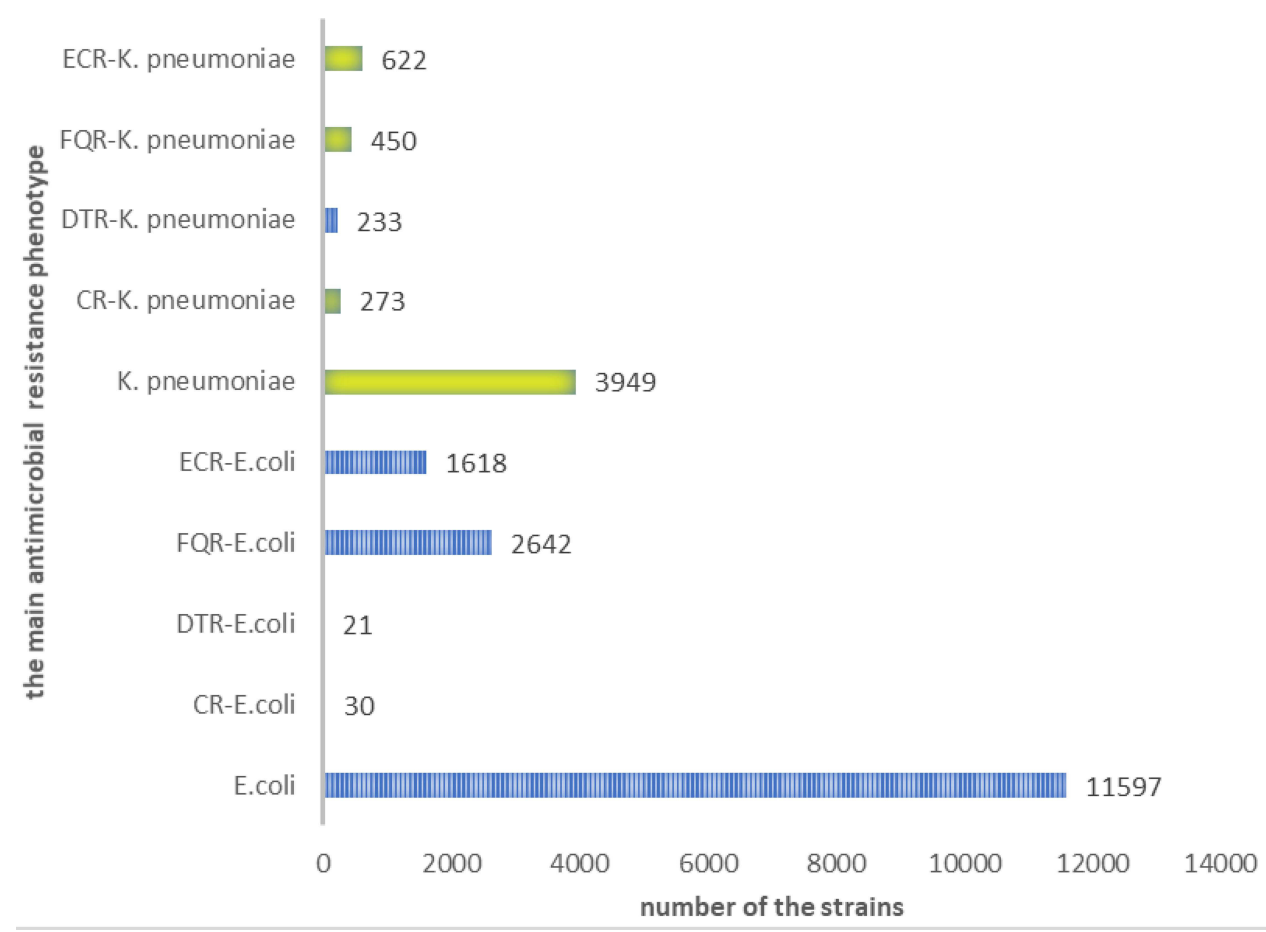

Figure 3 Distribution of the main antimicrobial resistance phenotypes.

Salmonella typhi. More and more studies showed that surveillance genomic data could accelerate understanding of virulence and antimicrobial resistance. ${ }^{29,30}$

Antibiotic susceptibility tests showed that the resistance rates of E. coli and $K$. pneumoniae to third-generation cefotaxime were significantly higher than those to ceftazidime, which is consistent with the 30-year data reported from CHINET in China. ${ }^{3}$ Wang et al showed that CTX-M was the most important ESBL type in China and that cefotaxime resistance might be a sign of ESBL-producing bacterial strains. ${ }^{31}$ E. coli and K. pneumoniae showed low resistance to amikacin, cefoperazone/sulbactam and imipenem; thus, these antibiotics might be used as empirical treatment options. Notably, in 2013-2017, the rates of K. pneumoniae resistance to imipenem and meropenem reached $15.8 \%$ and $17.5 \%$, respectively. Studies have confirmed that mortality rates of patients infected with carbapenem-resistant (CR) $K$. pneumoniae strains are significantly higher than those of patients infected with carbapenem-sensitive strains. ${ }^{32,33}$ CR $K$. pneumoniae strains often exhibit combined resistance to cephalosporins, fluoroquinolones, aminoglycosides, betalactamase inhibitors and other antimicrobial agents. ${ }^{34}$ Few antimicrobial agents, including tigecycline and polymyxin, can be used to treat CR K. pneumoniae. ${ }^{35} \mathrm{~K}$. pneumoniae resistant to colistin has emerged and colistin resistance was mainly associated with deleterious mutations and transposon in the mgrB gene. ${ }^{36}$ The resistance mechanism of Enterobacteriaceae to carbapenems is very complex. Producing carbapenemase including KPC, IMP, NDM-1, OXA48 and AmpC enzyme, or Extended-Spectrum $\beta$-Lactamases (ESBLs) including TEM, SHV, CTX may be the primary mechanisms. ${ }^{37}$ The study performed by Gao et al in our province showed that KPC-2 was the most common carbapenemase-resistant gene in K. pneumoniae to Carbapenems, whereas NDM-1 was more common in E. coli. ${ }^{37}$

This study revealed that $P$. aeruginosa and $A$. baumannii were the most common non-fermentative Gram-negative bacteria that caused BSIs. Susceptibility tests showed that resistance rates of $P$. aeruginosa to most antibiotics were less than $30 \%$. However, these results differed from those reported in a multicenter epidemiological study on the risk factors and clinical outcomes of nosocomial intra-abdominal infections in China (the Chinese antimicrobial resistance surveillance of nosocomial infections [CARES] 2007-2016), which indicated that $P$. aeruginosa showed high resistance to a variety of antimicrobial agents, except amikacin, whose susceptibility rate was $83.4 \%{ }^{38}$ The antimicrobial susceptibility profiles of $A$. baumannii isolates from BSIs were similar to those of $A$. baumannii isolates from abdominal infections. A. baumannii was alarmingly resistant to diverse antibiotics, including third-generation cephalosporins, 
aminoglycosides, fluoroquinolones and carbapenems. ${ }^{38}$ In this study, resistance rates of $A$. baumannii to common antibiotics increased significantly in 1998-2017. In 2003-2007, the antimicrobial resistance rate of A. baumannii was less than $50 \%$, but by $2013-2017$, the resistance rate reached $60-80 \%$. The emergence of multidrug-resistant A. baumannii, especially extensively drug-resistant and fully drug-resistant strains, has made clinical treatment difficult. A retrospective study from Oman indicated A. baumannii strains were highly resistant (50-83\%) to most of the tested antibiotics, with the highest against ceftriaxone (83\%) and ceftazidime $(75 \%)$, and lowest against colistin (1\%) and tigecycline (8\%). ${ }^{39}$ According to CLSI guidelines, S. maltophilia showed standard resistance levels to minocycline, levofloxacin and trimethoprim/sulfamethoxazole as determined by disk-diffusion tests, but MIC testing showed break points for ticarcillin/clavulanic acid, ceftazidime and chloramphenicol, minocycline, levofloxacin and trimethoprim/ sulfamethoxazole. ${ }^{13}$ Therefore, some hospitals could increase the antimicrobial sensitivity test results of some antibiotics after changing disk-diffusion tests to MIC tests. For example, for S. maltophilia, disk diffusion method had only three antimicrobial break points, while MIC method had six antimicrobial break points. As a result, clinicians had more choices in the empirical treatment. However, the disadvantage of the change of antimicrobial sensitivity test methodology was that the cumulative antimicrobial sensitivity data were inevitably biased when comparing data for many years. In this study, the resistance rate of S. maltophilia to ceftazidime increased to $58.1 \%$ in $2013-2017$, whereas the resistance rates of S. maltophilia to other antimicrobial agents were less than $25 \%$. Whether the increase in ceftazidime resistance was related to its wide clinical application requires further investigation and analysis.

Surveillance data on BSIs during 1998-2017 showed that the resistance rate of A. baumannii to common antibiotics has reached a high level, and the prevalence of CR K. pneumoniae has increased significantly, resulting in significant difficulties in clinical treatment. Our data show that vancomycin, teicoplanin, linezolid and trimethoprim/sulfamethoxazole can be used to treat MRSA. The resistance rate of MRSA to trimethoprim/sulfamethoxazole has decreased significantly, possibly related to the decreased of use of this antibiotics in recent years. Studies from China, South Korea and France have shown that the antimicrobial resistance rates of $S$. aureus, K. pneumoniae, E. coli, P. aeruginosa and Candida albicans also decreased with the decreased clinical use of these antimicrobial agents. ${ }^{40-43}$ Tigecycline and polymyxin can be used to empirically treat CR K. pneumoniae, E. coli and A. baumannii. ${ }^{44}$

This study had several limitations. The BSI incidence in Hubei Province was often reported from single research centers. We failed to find accurate data on the BSI incidence for all of Hubei Province during 1998-2017. Previous reports lacked demographic data. One shortcoming of this study was that the accurate BSI incidence was not calculated for Hubei Province. Another limitation was that different hospitals used different strain identification methods, including manual biochemical experiments and an IVD-MALDI Biotyper, and these results were undistinguishable once combined. Different hospitals adopted different antimicrobial sensitivity test methods, and the same hospital may change the antimicrobial sensitivity test method used between 1998 and 2017. Although each hospital strictly followed the CLSI guidelines, the inconsistency of test methods and the difference of antimicrobial sensitive consumables may lead to deviation in the analysis of antimicrobial resistance. The weakness of the analysis of the resistance mechanism involved in Gram-negative resistance to beta-lactams and more particularly to carbapenems was also a limitation of this study. We will increase the content of antimicrobial resistance mechanism research in the future.

\section{Conclusion}

CR K. pneumoniae, A. baumannii and MRSA present major challenges to controlling BSIs. S. typhi resistant to the third generation cephalosporins and quinolones has emerged, but the drug resistance rates were all less than 5\%. For K. pneumoniae DTR, CR, ECR and FQR were antimicrobial resistance phenotypes that could not be ignored while for E. coli DTR and CR were rare antimicrobial resistance phenotypes.

\section{Abbreviations}

BSI, Bacterial bloodstream infection; HBARSS, Hubei Province Antimicrobial Resistance Surveillance System; DTR, difficult-to-treat resistance; CR, Carbapenem-resistant; FQR, Fluoroquinolone resistant; ECR, Extended-spectrum cephalosporin resistant; CoNS, coagulase-negative staphylococcus; EARS-Net, European Antimicrobial Resistance Surveillance Network; CARES, Chinese antimicrobial Resistance surveillance of nosocomial Infections; MRSA, 
Methicillin-resistant S. aureus; CDC, Centers for Disease Control and Prevention; CLSI, Clinical Laboratory Standards Institute.

\section{Funding}

There is no funding to report.

\section{Disclosure}

The authors report no conflicts of interest in this work.

\section{References}

1. Ripabelli G, Salzo A, Mariano A, et al.; Collaborative Group for HPPSiMR. Healthcare-associated infections point prevalence survey and antimicrobials use in acute care hospitals (PPS 2016-2017) and long-term care facilities (HALT-3): a comprehensive report of the first experience in Molise Region, Central Italy, and targeted intervention strategies. J Infect Public Health. 2019;12(4):509-515. doi:10.1016/j.jiph.2019.01.060.

2. Shi D, Anwar TM, Pan H, et al. Genomic determinants of pathogenicity and antimicrobial resistance for 60 global listeria monocytogenes isolates responsible for invasive infections. Front Cell Infect Microbiol. 2021;11:718840. doi:10.3389/fcimb.2021.718840

3. Hu F, Zhu D, Wang F, Wang M. Current status and trends of antibacterial resistance in China. Clin Infect Dis. 2018;67(suppl_2):S128-S34. doi:10.1093/cid/ciy657

4. Hubei Statistical Service. Statistical yearbook of the Province; regional analytical report, Hubei Region—Hubei statistical service. Available from: www.tjj.hubei.gov.cn. Accessed September 29, 2019.

5. Iroh Tam PY, Musicha P, Kawaza K, et al. Emerging resistance to empiric antimicrobial regimens for pediatric bloodstream infections in Malawi (1998-2017). Clin Infect Dis. 2019;69(1):61-68. doi:10.1093/cid/ciy834

6. Musicha P, Cornick JE, Bar-Zeev N, et al. Trends in antimicrobial resistance in bloodstream infection isolates at a large urban hospital in Malawi (1998-2016): a surveillance study. Lancet Infect Dis. 2017;17(10):1042-1052. doi:10.1016/S1473-3099(17)30394-8

7. Liu Y, Cui BC, Pi CM, et al. Analysis of prognostic risk factors of bloodstream infections in Beijing communities: a retrospective study from 2015 to 2019. Mediterr J Hematol Infect Dis. 2021;13(1):e2021060. doi:10.4084/MJHID.2021.060

8. Van Boeckel TP, Gandra S, Ashok A, et al. Global antibiotic consumption 2000 to 2010: an analysis of national pharmaceutical sales data. Lancet Infect Dis. 2014;14(8):742-750. doi:10.1016/S1473-3099(14)70780-7

9. Tang X, Xiao M, Zhuo C, Xu Y, Zhong N. Multi-level analysis of bacteria isolated from inpatients in respiratory departments in China. $J$ Thorac Dis. 2018;10(5):2666-2675. doi:10.21037/jtd.2018.04.46

10. Health and Family Planning Commission. Operating procedures of blood culture for clinical microbiology laboratory. WS/T 503, GB, GBT, GB/T: Chinese Standard; 2017.

11. Weinstein MP, Murphy JR, Reller LB, Lichtenstein KA. The clinical significance of positive blood cultures: a comprehensive analysis of 500 episodes of bacteremia and fungemia in adults. II. Clinical observations, with special reference to factors influencing prognosis. Rev Infect Dis. 1983;5(1):54-70. doi:10.1093/clinids/5.1.54

12. Clinical and Laboratory Standards Institute. Analysis and Presentation of Cumulative Antimicrobial Susceptibility Test Data, M39-A4. Wayne, PA: Clin Lab Stand Institute; 2014.

13. Clinical and Laboratory Standards Institute. Performance Standards for Antimicrobial Susceptibility Testing, Thirtieth Informational Supplement, M100-S30. Wayne, PA: Clin Lab Stand Institute; 2020.

14. Gagliotti C, Balode A, Baquero F, et al. Escherichia coli and Staphylococcus aureus: bad news and good news from the European Antimicrobial Resistance Surveillance Network (EARS-Net, formerly EARSS), 2002 to 2009. Euro Surveill. 2011;16(11). doi:10.2807/ese.16.11.19819-en

15. Liu C, Xu M, Li X, et al. Trends in antimicrobial resistance in bloodstream infections at a large tertiary-care hospital in China: a 10-year retrospective study (2010-2019). J Glob Antimicrob Resist. 2021. doi:10.1016/j.jgar.2021.09.018

16. Tack B, Phoba MF, Van Puyvelde S, et al. Salmonella typhi from blood cultures in the Democratic Republic of the Congo: a 10-year surveillance. Clin Infect Dis. 2019;68(Suppl 2):S130-S137. doi:10.1093/cid/ciy1116

17. Crump JA, Sjolund-Karlsson M, Gordon MA, Parry CM. Epidemiology, clinical presentation, laboratory diagnosis, antimicrobial resistance, and antimicrobial management of invasive salmonella infections. Clin Microbiol Rev. 2015;28(4):901-937. doi:10.1128/CMR.00002-15

18. Buckle GC, Walker CL, Black RE. Typhoid fever and paratyphoid fever: systematic review to estimate global morbidity and mortality for 2010. J Glob Health. 2012;2(1):010401. doi:10.7189/jogh.02.010401

19. Al-Emran HM, Eibach D, Krumkamp R, et al. A multicountry molecular analysis of salmonella enterica serovar typhi with reduced susceptibility to ciprofloxacin in Sub-Saharan Africa. Clin Infect Dis. 2016;62(Suppl 1):S42-S46. doi:10.1093/cid/civ788

20. Crump JA, Mintz ED. Global trends in typhoid and paratyphoid fever. Clin Infect Dis. 2010;50(2):241-246. doi:10.1086/649541

21. Msemo OA, Mbwana J, Mahende C, et al. Epidemiology and antimicrobial susceptibility of salmonella enterica bloodstream isolates among febrile children in a rural district in Northeastern Tanzania: a cross-sectional study. Clin Infect Dis. 2019;68(Suppl 2):S177-S82. doi:10.1093/cid/ ciy1126

22. Cruz Espinoza LM, Nichols C, Adu-Sarkodie Y, et al. Variations of invasive salmonella infections by population size in Asante Akim North Municipal, Ghana. Clin Infect Dis. 2016;62(Suppl 1):S17-22. doi:10.1093/cid/civ787

23. Feasey NA, Dougan G, Kingsley RA, Heyderman RS, Gordon MA. Invasive non-typhoidal salmonella disease: an emerging and neglected tropical disease in Africa. Lancet. 2012;379(9835):2489-2499. doi:10.1016/S0140-6736(11)61752-2

24. Uche IV, MacLennan CA, Saul A, Systematic A. Review of the incidence, risk factors and case fatality rates of Invasive Nontyphoidal Salmonella (iNTS) disease in Africa (1966 to 2014). PLoS Negl Trop Dis. 2017;11(1):e0005118. doi:10.1371/journal.pntd.0005118

25. Crump JA, Heyderman RS. A perspective on invasive salmonella disease in Africa. Clin Infect Dis. 2015;61(Suppl 4):S235-40. doi:10.1093/cid/ civ709 
26. Kariuki S, Gordon MA, Feasey N, Parry CM. Antimicrobial resistance and management of invasive Salmonella disease. Vaccine. 2015;33(Suppl 3): C21-9. doi:10.1016/j.vaccine.2015.03.102

27. Kalonji LM, Post A, Phoba MF, et al. Invasive salmonella infections at multiple surveillance sites in the Democratic Republic of the Congo, 20112014. Clin Infect Dis. 2015;61(Suppl 4):S346-53. doi:10.1093/cid/civ713

28. Oneko M, Kariuki S, Muturi-Kioi V, et al. Emergence of community-acquired, multidrug-resistant invasive nontyphoidal salmonella disease in Rural Western Kenya, 2009-2013. Clin Infect Dis. 2015;61(Suppl 4):S310-6. doi:10.1093/cid/civ674

29. Qiu YF, Nambiar RB, Xu XB, et al. Global genomic characterization of Salmonella enterica Serovar Telelkebir. Front Microbiol. $2021 ; 12: 704152$. doi:10.3389/fmicb.2021.704152

30. Xu X, Chen Y, Pan H, et al. Genomic characterization of Salmonella uzaramo for human invasive infection. Microb Genom. 2020;6(7). doi:10.1099/mgen.0.000401

31. Wang P, Hu F, Xiong Z, et al. Susceptibility of extended-spectrum-beta-lactamase-producing Enterobacteriaceae according to the new CLSI breakpoints. J Clin Microbiol. 2011;49(9):3127-3131. doi:10.1128/JCM.00222-11

32. Falagas ME, Tansarli GS, Karageorgopoulos DE, Vardakas KZ. Deaths attributable to carbapenem-resistant Enterobacteriaceae infections. Emerg Infect Dis. 2014;20:1170-1175. doi:10.3201/eid2007.121004

33. Fraenkel-Wandel Y, Raveh-Brawer D, Wiener-Well Y, Yinnon AM, Assous MV. Mortality due to blaKPC Klebsiella pneumoniae bacteraemia. $J$ Antimicrob Chemother. 2016;71:1083-1087. doi:10.1093/jac/dkv414

34. Gutierrez-Gutierrez B, Salamanca E, de Cueto M, et al. Effect of appropriate combination therapy on mortality of patients with bloodstream infections due to carbapenemase-producing Enterobacteriaceae (INCREMENT): a retrospective cohort study. Lancet Infect Dis. 2017;17 (7):726-734. doi:10.1016/S1473-3099(17)30228-1

35. Tzouvelekis LS, Markogiannakis A, Psichogiou M, Tassios PT, Daikos GL. Carbapenemases in Klebsiella pneumoniae and other Enterobacteriaceae: an evolving crisis of global dimensions. Clin Microbiol Rev. 2012;25(4):682-707. doi:10.1128/CMR.05035-11

36. Di Tella D, Tamburro M, Guerrizio G, et al. Molecular epidemiological insights into colistin-resistant and carbapenemases-producing clinical Klebsiella pneumoniae Isolates. Infect Drug Resist. 2019;12:3783-3795. doi:10.2147/IDR.S226416

37. Gao B, Li X, Yang F, et al. Molecular epidemiology and risk factors of ventilator-associated pneumonia infection caused by carbapenem-resistant Enterobacteriaceae. Front Pharmacol. 2019;10:262. doi:10.3389/fphar.2019.00262

38. Zhang J, Zhao C, Chen H, et al. A multicenter epidemiology study on the risk factors and clinical outcomes of nosocomial intra-abdominal infections in China: results from the Chinese Antimicrobial Resistance Surveillance of Nosocomial Infections (CARES) 2007-2016. Infect Drug Resist. 2018;11:2311-2319. doi:10.2147/IDR.S182180

39. Sannathimmappa MB, Nambiar V, Aravindakshan R. Antibiotic resistance pattern of Acinetobacter baumannii Strains: a retrospective study from Oman. Saudi J Med Med Sci. 2021;9(3):254-260. doi:10.4103/sjmms.sjmms_855_20

40. Wang H, Wang H, Yu X, et al. Impact of antimicrobial stewardship managed by clinical pharmacists on antibiotic use and drug resistance in a Chinese hospital, 2010-2016: a retrospective observational study. BMJ Open. 2019;9(8):e026072. doi:10.1136/bmjopen-2018-026072

41. Zeng S, Xu Z, Wang X, et al. Time series analysis of antibacterial usage and bacterial resistance in China: observations from a tertiary hospital from 2014 to 2018. Infect Drug Resist. 2019;12:2683-2691. doi:10.2147/IDR.S220183

42. Kim B, Kim Y, Hwang H, et al. Trends and correlation between antibiotic usage and resistance pattern among hospitalized patients at university hospitals in Korea, 2004 to 2012: a nationwide multicenter study. Medicine. 2018;97(51):e13719. doi:10.1097/MD.0000000000013719

43. Abbara S, Pitsch A, Jochmans S, et al. Impact of a multimodal strategy combining a new standard of care and restriction of carbapenems, fluoroquinolones and cephalosporins on antibiotic consumption and resistance of Pseudomonas aeruginosa in a French intensive care unit. Int J Antimicrob Agents. 2019;53(4):416-422. doi:10.1016/j.ijantimicag.2018.12.001

44. Karakonstantis S, Kritsotakis EI, Gikas A. Treatment options for K. pneumoniae, P. aeruginosa and A. baumannii co-resistant to carbapenems, aminoglycosides, polymyxins and tigecycline: an approach based on the mechanisms of resistance to carbapenems. Infection. $2020 ; 48(6): 835-851$. doi:10.1007/s15010-020-01520-6

Infection and Drug Resistance

\section{Publish your work in this journal}

Infection and Drug Resistance is an international, peer-reviewed open-access journal that focuses on the optimal treatment of infection (bacterial, fungal and viral) and the development and institution of preventive strategies to minimize the development and spread of resistance. The journal is specifically concerned with the epidemiology of antibiotic resistance and the mechanisms of resistance development and diffusion in both hospitals and the community. The manuscript management system is completely online and includes a very quick and fair peer-review system, which is all easy to use. Visit http://www.dovepress.com/testimonials.php to read real quotes from published authors.

Submit your manuscript here: https://www.dovepress.com/infection-and-drug-resistance-journal 